Document downloaded from:

http://hdl.handle.net/10251/40399

This paper must be cited as:

Bonet Solves, JA.; WERNER RICKER; Ben (2011). Mean ergodic operators and reflexive Fréchet spaces. Proceedings of the Royal Society of Edinburgh: Section A Mathematics. 141:897-920.

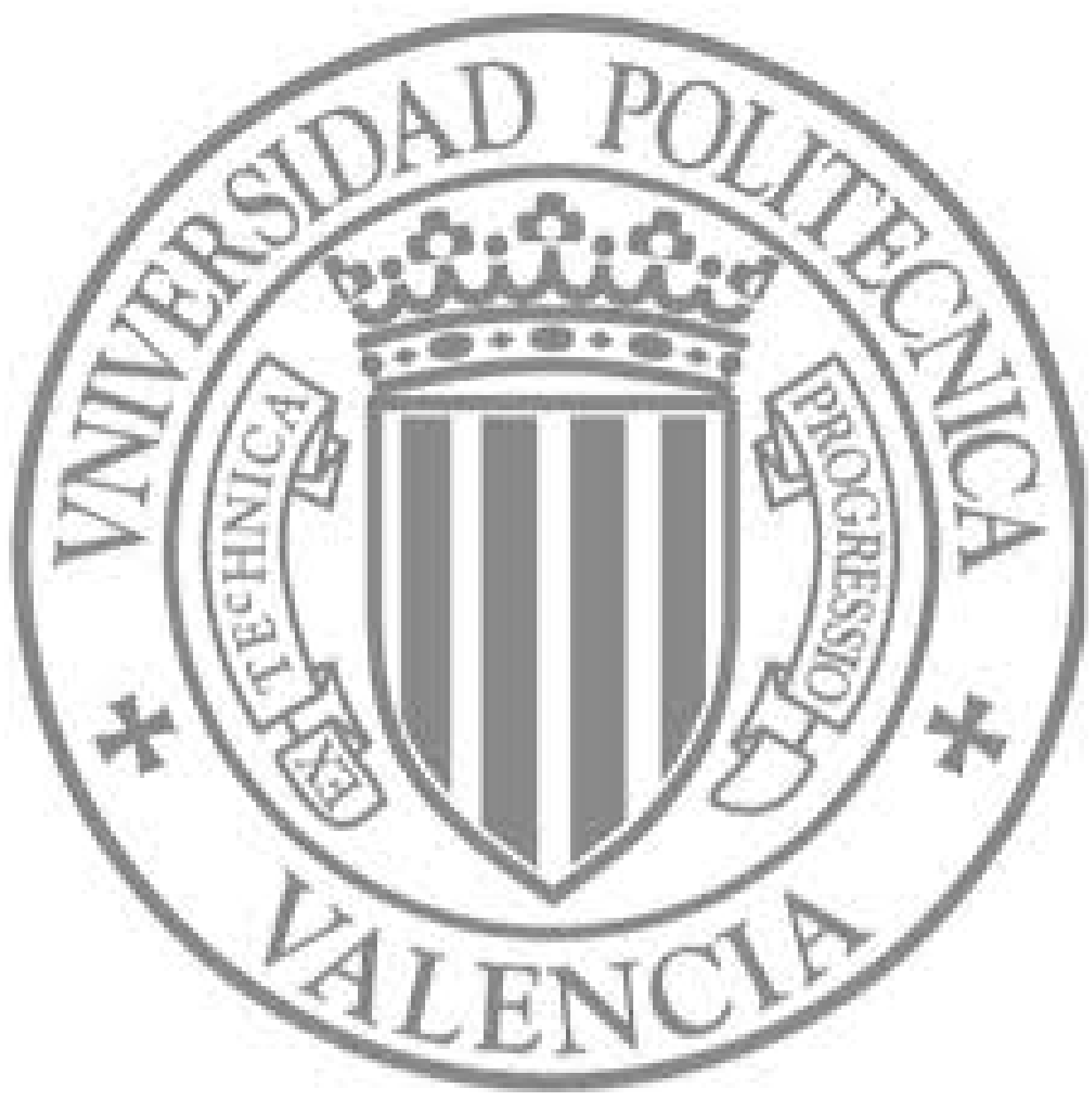

The final publication is available at

Copyright 


\title{
Mean ergodic operators and reflexive Fréchet lattices
}

\author{
José Bonet, Ben de Pagter ${ }^{\dagger}$ and Werner J. Ricker
}

\begin{abstract}
Connections between (positive) mean ergodic operators acting in Banach lattices and properties of the underlying lattice itself are well understood; see the works of Emel'yanov, Wolff and Zaharopol cited in the references. For Fréchet lattices (or more general locally convex solid Riesz spaces) there is virtually no information available. For a Fréchet lattice $E$, it is shown here (amongst other things) that every power bounded linear operator on $E$ is mean ergodic if and only if $E$ is reflexive if and only if $E$ is Dedekind $\sigma$-complete and every positive power bounded operator on $E$ is mean ergodic if and only if every positive power bounded operator in the strong dual $E_{\beta}^{\prime}$ (no longer a Fréchet lattice) is mean ergodic. An important technique is to develop criteria which detect when $E$ admits a (positively) complemented lattice copy of $c_{0}, \ell_{1}$ or $\ell_{\infty}$.
\end{abstract}

\section{Introduction and statement of results}

A continuous linear operator $T$ in a Banach space $E$ (or locally convex Hausdorff space, briefly lcHs) is called mean ergodic if the limits

$$
\lim _{n \rightarrow \infty} \frac{1}{n} \sum_{m=1}^{n} T^{m} x, \quad x \in E,
$$

exist (in the topology of $E$ ). J. von Neumann (1931) proved that unitary operators in Hilbert space are mean ergodic. Ever since, intensive research has been undertaken concerning mean ergodic operators and their applications; for the period up to the 1980's see [13], Ch. VIII, Section 4, [18], Ch. XVIII, [20], Ch. 2 , and the references therein.

It quickly became evident that there was an intimate connection between geometric properties of the underlying Banach space $E$ and mean ergodic operators on $E$. A continuous linear operator $T$ in $E$ (the space of all such operators is denoted by $\mathcal{L}(E)$ ) is called power bounded if $\sup _{m>0}\left\|T^{m}\right\|<\infty$. The space $E$ itself is called mean ergodic if for every power bounded $T \in \mathcal{L}(E)$ the limits

\footnotetext{
* Research partially supported by MEC and FEDER Project MTM 2007-62643; GV Project Prometeo 2008/101 and the net MTM 2007-30904-E (Spain)

†Support of the Alexander von Humboldt Foundation is gratefully acknowledged.

Math. Subject Classification (2000): Primary 46A04, 46A40, 47A35; Secondary 47B60, 47B 65

Key words and phrases: mean ergodic operator, power bounded, Fréchet lattice, locally solid Riesz space
} 
(1) exist. As a sample, F. Riesz (1938) showed that all $L_{p}$-spaces $(1<p<\infty)$ are mean ergodic. In 1938, E.R. Lorch proved that all reflexive Banach spaces are mean ergodic. In the opposite direction, in 2001 V.P. Fonf, M. Lin and P. Wojtaszczyk, [16], established (amongst other things) that a Banach space $E$ with a basis is reflexive if and only if $E$ is mean ergodic. For Banach lattices the requirement of a basis can be omitted. Indeed, in 1986, R. Zaharopol showed that if $E$ is a Dedekind $\sigma$-complete Banach lattice, then $E$ is reflexive if and only if every positive power bounded operator $T \in \mathcal{L}(E)$ is mean ergodic, [30]. For an arbitrary Banach lattice $E$, it was shown by E.Yu. Emel'yanov in 1997, [14], that $E$ is reflexive if and only if every regular power bounded operator $T \in \mathcal{L}(E)$ is mean ergodic (regular means that $T$ is the difference of two positive operators). According to E.Yu. Emel'yanov and M.P.H. Wolff, [15], a Banach lattice has order continuous norm if and only if every power order bounded operator on $E$ is mean ergodic.

For a lcHs $E$, the definition of $T \in \mathcal{L}(E)$ being mean ergodic (i.e., via (1)) makes perfectly good sense, as does the notion of power boundedness, now meaning that $\left\{T^{m}\right\}_{m>0}$ is an equicontinuous subset of $\mathcal{L}(E)$. The first "mean ergodic result" for (a special class of) power bounded operators $T$ on certain lcHs' $E$ is due to M. Altman, [6]. The restriction on $T$ that Altman imposed (a weak compactness condition) was later removed by K. Yosida ([28], Ch. VIII). In more recent times, most of the Banach space results mentioned above which connect geometric properties of the underlying space $E$ to mean ergodicity of operators acting on $E$ were extended to the Fréchet space setting in [1] and to more general lcHs' $E$ in [2], [3] and [8]. The aim of this article is to extend the above results concerned with mean ergodic operators in Banach lattices to the setting of Fréchet lattices. Classical examples of Fréchet lattices to keep in mind include the sequence space $\omega=\mathbb{R}^{\mathbb{N}}$ and all Köthe echelon spaces $\lambda_{p}(A)$ for $p \in\{0\} \cup[1, \infty]$, with $A$ a Köthe matrix relative to some countable index set (see e.g. [22]). We also mention $\ell^{p+}, 1 \leq p<\infty$ (see [23]) and $L^{p-}, 1<p<\infty$ (see [11]). Further examples are $L_{l o c}^{p}(\Omega)$ for $1 \leq p \leq \infty$ with $\Omega \subseteq \mathbb{R}^{N}$ open and $C(\Omega)$, equipped with the topology of uniform convergence on compact subsets of $\Omega$. Finally, if $m$ is any Fréchet space valued vector measure, then the spaces $L^{p}(m)$ (respectively, $L_{w}^{p}(m)$ ) consisting of the $p$-th power $m$-integrable (respectively, weakly $m$-integrable) functions are Fréchet lattices (see [9], [10], $[25])$.

So, let us formulate some of the main results. General references for the theory of lcHs' are [19], [22] and [26]. If $\Gamma_{E}$ is a system of continuous seminorms generating the topology of a lcHs $E$, then the strong operator topology $\tau_{s}$ in $\mathcal{L}(E)$ is determined by the seminorms

$$
q_{x}(S)=q(S x), \quad S \in \mathcal{L}(E),
$$

for each $x \in E$ and $q \in \Gamma_{E}$ (in which case we write $\mathcal{L}_{s}(E)$ ). The uniform operator topology $\tau_{b}$ in $\mathcal{L}(E)$ is defined via the seminorms

$$
q_{B}(S)=\sup _{x \in B} q(S x), \quad S \in \mathcal{L}(X)
$$

for each $q \in \Gamma_{E}$ and bounded set $B \subseteq E$ (in which case we write $\mathcal{L}_{b}(E)$ ). If $E$ is a Banach space, then $\tau_{b}$ is the operator norm topology on $\mathcal{L}(E)$. A Fréchet space is a complete, metrizable lcHs $E$, in which case $\Gamma_{E}$ may be taken 
countable. The topological dual space of a lcHs $E$ is denoted by $E^{\prime}$. The weak topology induced on $E$ by the pairing $\left\langle E, E^{\prime}\right\rangle$ is written as $\sigma\left(E, E^{\prime}\right)$ and the strong topology on $E$ (respectively $E^{\prime}$ ) is denoted by $\beta\left(E, E^{\prime}\right.$ ) (respectively $\beta\left(E^{\prime}, E\right)$ ), in which case we write $E_{\beta}$ (respectively $\left.E_{\beta}^{\prime}\right)$. Then, $E_{\beta}^{\prime}$ is called the strong dual of $E$ and $E^{\prime \prime}=\left(E_{\beta}^{\prime}\right)_{\beta}^{\prime}$ is the strong bidual of $E$. If $E=E^{\prime \prime}$ as vector spaces (respectively, additionally topologically), then $E$ is called semireflexive (respectively, reflexive). For a Fréchet space $E$, the strong dual $E_{\beta}^{\prime}$ need not be metrizable but, $E^{\prime \prime}$ is again a Fréchet space containing $E$ as a closed subspace ([22], Corollary 25.10).

Relevant references for the theory of Riesz spaces (which will always be over $\mathbb{R})$, with $\leq$ denoting the order, are [21], [24], [29]. For locally convex-solid Riesz spaces $E$ (briefly lc-solid Riesz spaces) we refer to [4], [5], [17], for example. In this case, the seminorms $q \in \Gamma_{E}$ can all be chosen to be Riesz seminorms, that is, $q(x) \leq q(y)$ whenever $|x| \leq|y|$ in $E$ ([4], Theorem 6.1). As usual, a linear operator $T \in \mathcal{L}(E)$ is called positive if $T x \geq 0$ whenever $x \in E^{+}$, where $E^{+}=\{x \in E: x \geq 0\}$ is the positive cone of $E$. A Riesz space $E$ is said to be Dedekind $(\sigma-)$ complete if every non-empty (countable) subset of $E$ that is order bounded from above has a supremum. Typical examples of Riesz spaces which fail to be Dedekind $\sigma$-complete are the sequence space $c$ and the space of continuous functions $C([0,1])$.

A lc-solid Riesz space which is metrizable and complete is simply called a Fréchet lattice ([4], p. 111).

Theorem 1.1 If $E$ is a Fréchet lattice, then the following assertions are equivalent.

(i) E is reflexive.

(ii) $E$ is mean ergodic.

(iii) $E$ is Dedekind $\sigma$-complete and every positive power bounded operator on $E$ is mean ergodic.

(iv) Every positive, power bounded operator on $E_{\beta}^{\prime}$ is mean ergodic.

The main tool needed to establish Theorem 1.1 is of interest in its own right. Two lc-solid Riesz spaces $E$ and $F$ are called Riesz homeomorphic if there exists a Riesz homeomorphism $J: E \rightarrow F$ (that is, $J$ is a linear lattice homomorphism from $E$ onto $F$ which is also a homeomorphism). If $E$ contains a Riesz subspace which is Riesz homeomorphic to $F$, then we say that $E$ contains a lattice copy of $F$. As usual, a closed Riesz subspace $F$ of a lc-solid Riesz space $E$ is said to be (positively) complemented in $E$ if $F$ is the range of a linear continuous (positive) projection.

Crucial for establishing Theorem 1.1 is the following result.

Theorem 1.2 If $E$ is a Dedekind $\sigma$-complete lc-solid Riesz space which is complete and $\aleph_{0}$-barrelled, then the following assertions are equivalent.

(i) E is not semireflexive.

(ii) E contains a lattice copy of either $\ell_{\infty}, \ell_{1}$ or $c_{0}$. 
The relevance of the Banach lattices $c_{0}, \ell_{1}$ and $\ell_{\infty}$ is that each one admits a positive power bounded operator which fails to be mean ergodic. Indeed, denoting the elements in these sequence spaces by $x=\left(x_{1}, x_{2}, \ldots\right)$, it can be verified that the operators

$$
\begin{aligned}
& T_{0}: x \longmapsto\left(x_{1}, x_{1}, x_{2}, x_{3}, \ldots\right), \quad x \in c_{0}, \\
& T_{1}: x \longmapsto\left(0, x_{1}, x_{2}, x_{3}, \ldots\right), \quad x \in \ell_{1}, \\
& T_{\infty}: x \longmapsto\left(x_{2}, x_{3}, x_{4}, \ldots\right), \quad x \in \ell_{\infty},
\end{aligned}
$$

on the spaces $c_{0}, \ell_{1}$ and $\ell_{\infty}$ respectively, have the stated properties.

Given a Riesz space $E$, a linear map $T: E \rightarrow E$ is called power order bounded if, for every $x \in E^{+}$there exists $z \in E^{+}$such that

$$
\bigcup_{m=0}^{\infty} T^{m}([-x, x]) \subseteq[-z, z],
$$

where $[-u, u]$ denotes the order interval $\{y \in E:-u \leq y \leq u\}$, for each $u \in E^{+}$. Order intervals in a lc-solid Riesz space $E$ are always topologically bounded ([4], Theorem 5.4). Hence, if $E$ is barrelled and $T \in \mathcal{L}(E)$ is power order bounded, then the uniform boundedness principle implies that $T$ is power bounded ([22], Proposition 23.27).

Recall that a lc-solid Riesz space $E$ has a $(\sigma-)$ Lebesgue topology if, for every decreasing (sequence) net $x_{\alpha} \downarrow_{\alpha} 0$ it follows that $x_{\alpha} \rightarrow_{\alpha} 0$ with respect to the given topology ([4], p. 52). For Banach lattices this notion corresponds to $(\sigma-)$ order continuity of the norm ([24], Ch. 2, Section 4). The extension of the above mentioned result of Emelyanov and Wolff can now be formulated.

Theorem 1.3 For a Fréchet lattice E the following assertions are equivalent.

(i) E has a Lebesgue topology.

(ii) Every power order bounded operator on $E$ is mean ergodic.

We mention that Theorems 1.1-1.3 will actually be established in somewhat more generality than the (more transparent) versions formulated above.

For a Banach space $E$ with a basis it is known that $E$ is uniformly mean ergodic if and only if $E$ is finite dimensional ([16], Corollary 3). Here, a lcHs $E$ is called uniformly mean ergodic if every power bounded operator $T$ on $E$ has the property that its Cesàro means

$$
T_{[n]}=\frac{1}{n} \sum_{m=1}^{n} T^{m}, \quad n \in \mathbb{N},
$$

form a convergent sequence in $\mathcal{L}_{b}(E)$. For Banach lattices the requirement of a basis can be omitted.

Theorem 1.4 A Banach lattice $E$ is uniformly mean ergodic if and only if $E$ is finite dimensional.

It is known that every Montel Fréchet lattice (e.g. $\omega$ or $\lambda_{p}(A), 1 \leq p \leq \infty$, for those Köthe matrices $A$ such that $\lambda_{1}(A)$ is reflexive; [22], Theorem 27.9) is necessarily uniformly mean ergodic ([1], Proposition 2.8). Our final result may be viewed as an analogue of Theorem 1.4 for non-normable Fréchet lattices. We point out that every Montel Fréchet lattice is necessarily discrete ([4], Corollary 21.13). 
Theorem 1.5 A Fréchet lattice $E$ is Montel if and only if $E$ is discrete and uniformly mean ergodic.

\section{Some preliminary results}

For a Riesz space $E$, we recall that the order dual $E^{\sim}$ is always a Dedekind complete Riesz space ([4], Theorem 3.3). A classical result of F. Riesz states that in any Dedekind complete Riesz space $E$, every band $B$ is a projection band, that is, $E=B \oplus B^{d}$ or, equivalently, there exists a linear projection $P: E \rightarrow E$ with range $\operatorname{Im}(\mathrm{P})=\mathrm{B}$ and satisfying $P x \in[0, x], x \in E^{+}$([4], Theorem 2.12; [24], Theorem 1.2.9). Such a projection $P$ is called a band projection in $E$ (note that if $E$ is a lc-solid Riesz space, then every band projection $P$ is continuous, because $|P x| \leq|x|$ for $x \in E)$. Here, $B^{d}=\{x \in E:|x| \wedge|y|=0, \forall y \in B\}$.

If $E$ is a lc-solid Riesz space, then $E_{\beta}^{\prime}$ is also a lc-solid Riesz space whose topology is given by the family of Riesz seminorms

$$
q_{B}\left(x^{\prime}\right)=\sup _{x \in B}\left|\left\langle x, x^{\prime}\right\rangle\right|, \quad x^{\prime} \in E_{\beta}^{\prime},
$$

as $B$ runs through the collection $\mathcal{B}_{s}$ of all bounded, solid subsets of $E$ ([4], pp. $59,129)$. Moreover, $E_{\beta}^{\prime}$ is an ideal in $E^{\sim}$ and so, in particular, $E_{\beta}^{\prime}$ is Dedekind complete ([4], Theorem 5.7). If $E$ happens to be barrelled, then $E_{\beta}^{\prime}$ is a band in $E^{\sim}$ ([4], Theorem 6.4). Consequently, $E_{\beta}^{\prime}$ is then topologically complete ([4], Theorem 19.13).

We will require the following result on extending linear functionals ([29], Theorem 83.17).

Theorem 2.1 Let $E$ be a Riesz space, $F \subseteq E$ be a Riesz subspace (i.e., vector sublattice) and $\theta: E \rightarrow \mathbb{R}$ be a sublinear functional which is absolute (i.e., $\theta(x)=\theta(|x|), x \in E$ ) and monotone on $E^{+}$(i.e., $\theta(x) \leq \theta(y)$ whenever $0 \leq x \leq y$ in $E$ ). If $\varphi: F \rightarrow \mathbb{R}$ is a positive linear functional satisfying $|\langle x, \varphi\rangle| \leq \theta(x)$ for $x \in F$, then there exists a positive linear functional $\psi: E \rightarrow$ $\mathbb{R}$ such that $\left.\psi\right|_{F}=\varphi$ and $|\langle x, \psi\rangle| \leq \theta(x)$ for $x \in E$.

As an immediate application, we present a result which is well known in the Banach lattice setting ([24], Proposition 2.3.11).

Proposition 2.2 If $F$ is a lattice copy of $\ell_{1}$ in a lc-solid Riesz space $E$, then

(i) $F$ is positively complemented in $E$, and

(ii) $E_{\beta}^{\prime}$ contains a lattice copy of $\ell_{\infty}$.

Proof. (i). Let $\|\cdot\|_{1}$ be a Riesz norm on $F$ such that the topology of $F$ induced by $E$ is given by $\|\cdot\|_{1}$ and $\left(F,\|\cdot\|_{1}\right)$ is Riesz isometric to $\ell_{1}$. In particular, there exists a continuous Riesz seminorm $r$ on $E$ such that

$$
\|x\|_{1} \leq r(x), \quad x \in F .
$$

Let $\left\{v_{n}\right\}_{n=1}^{\infty} \subseteq F$ correspond to the standard unit basis vectors of $\ell_{1}$ (so that $\left\|v_{n}\right\|_{1}=1$ for all $\left.n \in \mathbb{N}\right)$. 
For each $x \in F$ there exists a unique sequence $\left\{\alpha_{n}(x)\right\}_{n=1}^{\infty} \in \ell_{1}$ satisfying $x=\sum_{n=1}^{\infty} \alpha_{n}(x) v_{n}$, with the series convergent in $\left(F,\|\cdot\|_{1}\right)$. Note that $x \in F^{+}$ if and only if $\alpha_{n}(x) \geq 0$ for all $n \in \mathbb{N}$. Since $\left|\alpha_{n}(x)\right| \leq\|x\|_{1} \leq r(x)$ for $x \in F$, it is clear that $\alpha_{n} \in\left(F^{\prime}\right)^{+}$for all $n \in \mathbb{N}$, where $\left\langle x, \alpha_{n}\right\rangle=\alpha_{n}(x), x \in F$. Define the positive linear functional $y_{1}^{\prime} \in F^{\prime}$ by setting

$$
\left\langle x, y_{1}^{\prime}\right\rangle=\sum_{k=1}^{\infty}\left\langle x, \alpha_{k}\right\rangle, \quad x \in F,
$$

in which case

$$
\left|\left\langle x, y_{1}^{\prime}\right\rangle\right| \leq\left\langle|x|, y_{1}^{\prime}\right\rangle \leq r(|x|)=r(x), \quad x \in F .
$$

Evidently, $0 \leq \alpha_{n} \leq y_{1}^{\prime}$ for all $n \in \mathbb{N}$. By Theorem 2.1, applied to $\theta=r$ and $\varphi=y_{1}^{\prime}$, there exists $0 \leq x_{1}^{\prime} \in E^{\sim}$ with $\left.x_{1}^{\prime}\right|_{F}=y_{1}^{\prime}$ such that $\left|\left\langle x, x_{1}^{\prime}\right\rangle\right| \leq r(x)$, $x \in E$. In particular, $x_{1}^{\prime} \in\left(E^{\prime}\right)^{+}$. Since, for each $n \in \mathbb{N}$,

$$
\left|\left\langle x, \alpha_{n}\right\rangle\right| \leq\left\langle|x|, \alpha_{n}\right\rangle \leq\left\langle|x|, y_{1}^{\prime}\right\rangle=\left\langle|x|, x_{1}^{\prime}\right\rangle, \quad x \in F,
$$

it follows from Theorem 2.1 that there exists $0 \leq z_{n}^{\prime} \in E^{\sim}$ with $\left.z_{n}^{\prime}\right|_{F}=\alpha_{n}$ such that $\left|\left\langle x, z_{n}^{\prime}\right\rangle\right| \leq\left\langle|x|, x_{1}^{\prime}\right\rangle$ for $x \in E$. In particular, $z_{n}^{\prime} \in\left(E^{\prime}\right)^{+}$and $0 \leq z_{n}^{\prime} \leq x_{1}^{\prime}$ for all $n \in \mathbb{N}$. Let $\varphi_{n}$ be the minimal positive extension of the restriction of $z_{n}^{\prime}$ to the principal ideal $E_{v_{n}}$ generated by $v_{n}$ in $E$ (see [29], Theorems 83.7 and 83.8). It follows from $0 \leq \varphi_{n} \leq z_{n}^{\prime}$ that $\varphi_{n} \in\left(E^{\prime}\right)^{+}$and $0 \leq \varphi_{n} \leq x_{1}^{\prime}$ for all $n$. Since $\left\langle v_{m}, \varphi_{n}\right\rangle=\delta_{n, m}$ for all $n, m \in \mathbb{N}$, it is clear that $\left.\varphi_{n}\right|_{F}=\alpha_{n}$. Furthermore, since $v_{n} \wedge v_{m}=0(n \neq m)$, it can be verified (see [5], Exercise 2.3) that $\varphi_{n} \wedge \varphi_{m}=0$ in $E^{\prime}$ whenever $n \neq m$.

If $x \in E$, then

$$
\sum_{k=1}^{n}\left|\left\langle x, \varphi_{k}\right\rangle\right| \leq \sum_{k=1}^{n}\left\langle|x|, \varphi_{k}\right\rangle=\left\langle|x|, \bigvee_{k=1}^{n} \varphi_{k}\right\rangle \leq\left\langle|x|, x_{1}^{\prime}\right\rangle
$$

for all $n \in \mathbb{N}$ and so, $\sum_{k=1}^{\infty}\left|\left\langle x, \varphi_{k}\right\rangle\right|<\infty$. Consequently,

$$
\sum_{n=1}^{\infty}\left\|\left\langle x, \varphi_{n}\right\rangle v_{n}\right\|_{1}=\sum_{n=1}^{\infty}\left|\left\langle x, \varphi_{n}\right\rangle\right|<\infty, \quad x \in E .
$$

Hence, the series

$$
P x=\sum_{n=1}^{\infty}\left\langle x, \varphi_{n}\right\rangle v_{n}, \quad x \in E,
$$

converges in the complete space $F$. It is now clear that $P$ is a positive projection in $E$ onto $F$.

(ii). Using the notation introduced in the proof of (i), define the map $\Phi_{0}$ : $\ell_{\infty}^{+} \rightarrow\left(E^{\prime}\right)^{+}$by setting

$$
\Phi_{0}(\lambda)=\bigvee_{n=1}^{\infty} \lambda_{n} \varphi_{n}, \quad 0 \leq \lambda=\left(\lambda_{n}\right) \in \ell_{\infty}^{+} .
$$

Since $0 \leq \varphi_{n} \leq x_{1}^{\prime}$ for all $n$ and $E^{\prime}$ is Dedekind complete, this map is well defined and satisfies $0 \leq \Phi_{0}(\lambda) \leq\|\lambda\|_{\infty} x_{1}^{\prime}$ for $\lambda \in \ell_{\infty}^{+}$. Since $\left\{\varphi_{n}\right\}_{n=1}^{\infty}$ is a disjoint system in $\left(E^{\prime}\right)^{+}$, it is clear that $\Phi_{0}$ is additive, positive homogeneous and that $\Phi_{0}(\lambda) \wedge \Phi_{0}(\mu)=0$ whenever $\lambda \wedge \mu=0$ in $\ell_{\infty}^{+}$. Therefore, $\Phi_{0}$ has a unique extension to a Riesz homomorphism $\Phi: \ell_{\infty} \rightarrow E^{\prime}$ ([4], Theorem 1.17 
and Lemma 3.1). We claim that $\Phi$ is a linear homeomorphism from $\ell_{\infty}$ onto its range in $E_{\beta}^{\prime}$. Indeed, if $p$ is any Riesz seminorm on $E^{\prime}$, then

$$
p(\Phi(\lambda))=p(\Phi(|\lambda|)) \leq\|\lambda\|_{\infty} p\left(x_{1}^{\prime}\right), \quad \lambda \in \ell_{\infty} .
$$

On the other hand, if $B$ is the convex solid hull in $E$ of the bounded set $\left\{v_{n}\right\}_{n=1}^{\infty}$, then the continuous Riesz seminorm $q_{B}$ on $E_{\beta}^{\prime}$, defined by $(6)$, satisfies $q_{B}\left(\varphi_{n}\right)=$ 1 for all $n \in \mathbb{N}$. If $\lambda=\left(\lambda_{n}\right) \in \ell_{\infty}$, then

$$
|\Phi(\lambda)|=\Phi(|\lambda|) \geq\left|\lambda_{n}\right| \varphi_{n}
$$

and so, $q_{B}(\Phi(\lambda)) \geq\left|\lambda_{n}\right|$ for all $n \in \mathbb{N}$. This implies that $q_{B}(\Phi(\lambda)) \geq\|\lambda\|_{\infty}$, $\lambda \in \ell_{\infty}$, and we may conclude that $\Phi$ is a linear homeomorphism. The proof is complete.

Remark 2.3 (a) It can be verified that the adjoint $P^{\prime} \in \mathcal{L}\left(E_{\beta}^{\prime}\right)$ of the projection $P$ in $E$ defined by (7) is a positive projection in $E_{\beta}^{\prime}$ onto the lattice copy $\Phi\left(\ell_{\infty}\right)$ of $\ell_{\infty}$ in $E_{\beta}^{\prime}$, as constructed in (ii) of the above proof.

(b) Any lattice copy of $\ell_{\infty}$ in a lc-solid Riesz space $E$ is positively complemented. Indeed, suppose that $F$ is a Riesz subspace of $E$ and let $J: \ell_{\infty} \rightarrow F$ be a Riesz homeomorphism. For every continuous Riesz seminorm $p$ on $E$ there exists a constant $C_{p} \geq 0$ such that $p(J \lambda) \leq C_{p}\|\lambda\|_{\infty}$ for $\lambda \in \ell_{\infty}$. There also exists a continuous Riesz seminorm $q$ on $E$ such that $\|\lambda\|_{\infty} \leq q(J \lambda)$ for $\lambda \in \ell_{\infty}$. For each $n \in \mathbb{N}$, define the positive linear functional $\varphi_{n}$ on $F$ by $\left\langle x, \varphi_{n}\right\rangle=\left(J^{-1} x\right)(n), x \in F$, where $\left(J^{-1} x\right)(n)$ denotes the $n$-th coordinate of $J^{-1} x$. Note that $J^{-1} x=\left(\left\langle x, \varphi_{n}\right\rangle\right)$ and hence, $J\left(\left(\left\langle x, \varphi_{n}\right\rangle\right)\right)=x$ for all $x \in F$. Since

$$
\left|\left\langle x, \varphi_{n}\right\rangle\right|=\left|\left(J^{-1} x\right)(n)\right| \leq\left\|J^{-1} x\right\|_{\infty} \leq q(x), \quad x \in F,
$$

it follows from Theorem 2.1 that, for each $n \in \mathbb{N}$, there exists a positive linear functional $\psi_{n}$ on $E$ such that $\left.\psi_{n}\right|_{F}=\varphi_{n}$ and $\left|\left\langle x, \psi_{n}\right\rangle\right| \leq q(x)$, $x \in E$ (and so, $0 \leq \psi_{n} \in E^{\prime}$ ). It is clear that $\left(\left\langle x, \psi_{n}\right\rangle\right) \in \ell_{\infty}$ for all $x \in E$. Defining the map $P: E \rightarrow E$ via

$$
P x=J\left(\left(\left\langle x, \psi_{n}\right\rangle\right)\right), \quad x \in E,
$$

it can be checked that $P$ is a positive continuous projection in $E$ onto $F$. This proves the claim.

Proposition 2.4 Suppose that E is a sequentially complete, lc-solid Riesz space, with the property that countable, bounded subsets of $E_{\beta}^{\prime}$ are equicontinuous. If $E_{\beta}^{\prime}$ contains a lattice copy of $c_{0}$, then $E$ contains a positively complemented lattice copy of $\ell_{1}$.

Remark 2.5 (i) Every $\aleph_{0}$-barrelled lcHs $E$ has the property that countable, bounded subsets of $E_{\beta}^{\prime}$ are equicontinuous ([26], Observation 8.2.2 (a)). All barrelled (hence, all Fréchet) lcHs' are $\aleph_{0}$-barrelled ([26], Observation 8.2.2 (b)); the same is true for all complete $(D F)$-spaces ([26], Observation 8.2 .2 (c)), which include the strong duals of Fréchet spaces. 
(ii) For E a Banach lattice, Proposition 2.4 occurs in [24], Proposition 2.3.12.

Proof. (of Proposition 2.4) The idea of the proof follows the lines of that of implication (iii) $\Rightarrow$ (i) in [24], Proposition 2.3.12, with various modifications required due to the new setting.

Let $F$ be a lattice copy of $c_{0}$ in $E_{\beta}^{\prime}$ and let $\left\{x_{n}^{\prime}\right\}_{n=1}^{\infty} \subseteq F$ correspond to the standard unit basis vectors of $c_{0}$, in which case $x_{n}^{\prime} \geq 0, n \in \mathbb{N}$. Then $\left\{x_{n}^{\prime}\right\}_{n=1}^{\infty}$ is a bounded subset of $E_{\beta}^{\prime}$ which is not a null sequence. Hence, there exists a set $B \in \mathcal{B}_{s}$ such that $q_{B}\left(x_{n}^{\prime}\right) \nrightarrow 0$ as $n \rightarrow \infty$, with $q_{B}$ given by (6). So, by passing to a subsequence if necessary, there exists $\delta>0$ such that $q_{B}\left(x_{n}^{\prime}\right) \geq \delta, n \in \mathbb{N}$. It follows from (6) that there exists a sequence $\left\{x_{n}\right\}_{n=1}^{\infty} \subseteq B$ satisfying

$$
\left|\left\langle x_{n}, x_{n}^{\prime}\right\rangle\right| \geq \delta / 2, \quad n \in \mathbb{N}
$$

Since $\left|\left\langle x, x_{n}^{\prime}\right\rangle\right| \leq\left\langle|x|, x_{n}^{\prime}\right\rangle, x \in E$, it is clear from (8) that $\left\langle\left|x_{n}\right|, x_{n}^{\prime}\right\rangle \geq \delta / 2$ for all $n \in \mathbb{N}$, with $\left\{\left|x_{n}\right|\right\}_{n=1}^{\infty} \subseteq B$ (as $B$ is solid). Accordingly, replacing $x_{n}$ by $\left|x_{n}\right|$, we may assume that $\left\{x_{n}\right\}_{n=1}^{\infty} \subseteq B^{+}$. Moreover, since $\left\{x_{n}^{\prime}\right\}_{n=1}^{\infty}$ and $\left\{x_{n}\right\}_{n=1}^{\infty}$ are bounded in $E_{\beta}^{\prime}$ and $E$, respectively, there exists a constant $C>0$ such that $\left\langle x_{n}, x_{n}^{\prime}\right\rangle \leq C$ for all $n \in \mathbb{N}$, that is,

$$
\delta / 2 \leq\left\langle x_{n}, x_{n}^{\prime}\right\rangle \leq C, \quad n \in \mathbb{N} .
$$

By replacing $x_{n}$ with $(4 / \delta) x_{n}, B$ with $(4 / \delta) B$ and $C$ with $(4 / \delta) C$, we may assume that the sequence $\left\{x_{n}\right\}_{n=1}^{\infty}$ satisfies

$$
2 \leq\left\langle x_{n}, x_{n}^{\prime}\right\rangle \leq C, \quad n \in \mathbb{N} .
$$

Since $\left\{x_{n}\right\}_{n=1}^{\infty}$ is bounded, given any continuous Riesz seminorm $p$ on $E$, we have $\sup _{n} p\left(x_{n}\right)<\infty$ and hence, $\sum_{n=1}^{\infty} p\left(2^{-n} x_{n}\right)<\infty$. By the sequential completeness of $E$, it follows that there exists $e \in E^{+}$such that $\sum_{n=1}^{\infty} 2^{-n} x_{n}=e$, as a convergent series in $E$. Consequently, $\left\{x_{n}\right\}_{n=1}^{\infty}$ is contained in the principal ideal $E_{e}$ generated by $e$ in $E$. Applying [24], Theorem 2.3.1, to the principal ideal $E_{e}$ and the sequences $\left\{x_{n}\right\}_{n=1}^{\infty}$ and $\left\{\left.x_{n}^{\prime}\right|_{E_{e}}\right\}_{n=1}^{\infty}$, and passing to a subsequence if necessary, it follows that there exists a pairwise disjoint sequence $\left\{v_{n}\right\}_{n=1}^{\infty}$ in $E^{+}$such that

$$
0 \leq v_{n} \leq x_{n}, \quad \text { and } \quad\left\langle v_{n}, x_{n}^{\prime}\right\rangle \geq 1, \quad n \in \mathbb{N} .
$$

Since $B$ is solid, it is clear that $\left\{v_{n}\right\}_{n=1}^{\infty} \subseteq B^{+}$.

Define the countable set $A \subseteq\left(E^{\prime}\right)^{+}$by

$$
A=\left\{\sum_{j=1}^{n} x_{j}^{\prime}: n \in \mathbb{N}\right\}
$$

Since $A$ is bounded in $F \cong c_{0}$, it is also bounded in $E_{\beta}^{\prime}$. By hypothesis, $A$ is then equicontinuous. Consequently, there exists a continuous Riesz seminorm $p_{0}$ on $E$ such that

$$
\left|\left\langle x, x^{\prime}\right\rangle\right| \leq p_{0}(x), \quad x \in E, \quad x^{\prime} \in A .
$$

Fix $a=\left(a_{1}, \ldots, a_{n}, 0,0, \ldots\right) \in c_{00}$. The elements $\left\{v_{j}\right\}_{j=1}^{n}$ are pairwise disjoint and so $\left|\sum_{j=1}^{n} a_{j} v_{j}\right|=\sum_{j=1}^{n}\left|a_{j}\right| v_{j}$. Since $\sum_{j=1}^{n} x_{j}^{\prime} \in A$, it follows from (10) and 
(9) that

$$
\begin{aligned}
p_{0}\left(\sum_{j=1}^{n} a_{j} v_{j}\right) & =p_{0}\left(\sum_{j=1}^{n}\left|a_{j}\right| v_{j}\right) \geq\left\langle\sum_{j=1}^{n}\left|a_{j}\right| v_{j}, \sum_{k=1}^{n} x_{k}^{\prime}\right\rangle \\
& \geq \sum_{j=1}^{n}\left|a_{j}\right|\left\langle v_{j}, x_{j}^{\prime}\right\rangle \geq \sum_{j=1}^{n}\left|a_{j}\right|=\|a\|_{1} .
\end{aligned}
$$

This shows that

$$
\|a\|_{1} \leq p_{0}\left(\sum_{j=1}^{n} a_{j} v_{j}\right) .
$$

On the other hand, given any continuous Riesz seminorm $p$ on $E$, we have

$$
p\left(\sum_{j=1}^{n} a_{j} v_{j}\right) \leq C_{p}\|a\|_{1},
$$

where $C_{p}=\sup _{k \in \mathbb{N}} p\left(v_{k}\right)<\infty$, as $\left\{v_{k}\right\}_{k=1}^{\infty} \subseteq B$ is bounded. Estimates (11) and (12) suffice to conclude that the closed Riesz subspace generated by $\left\{v_{n}\right\}_{n=1}^{\infty}$ is Riesz homeomorphic to $\ell_{1}$.

The conclusion now follows from Proposition 2.2 (i).

For a Banach space $E$ it is a classical result of $\mathrm{C}$. Bessaga and A. Pelczynski that the dual Banach space $E_{\beta}^{\prime}$ contains an isomorphic copy of the Banach space $c_{0}$ if and only if $E$ contains a complemented copy of $\ell_{1}$ ([12], p. 48). The extension of this result to Fréchet spaces can be found in [7], Lemma 10. The following corollary, which is an immediate consequence of Proposition 2.2 (ii) and Proposition 2.4, may be considered as a lattice version of these results.

Corollary 2.6 If $E$ is a sequentially complete lc-solid Riesz space with the property that countable, bounded subsets of $E_{\beta}^{\prime}$ are equicontinuous, then $E_{\beta}^{\prime}$ contains a lattice copy of $c_{0}$ if and only if $E$ contains a (positively complemented) lattice copy of $\ell_{1}$.

Corollary 2.6 is known for Banach lattices; see Propositions 2.3.11 and 2.3.12 in [24].

The following simple fact will be required in the sequel. Recall that the topology in a locally solid Riesz space $E$ is said to be pre-Lebesgue whenever every increasing, order bounded sequence in $E^{+}$is Cauchy (see [4], Definition 8.1).

Lemma 2.7 Let $E$ be a lc-solid Riesz space with a pre-Lebesgue topology. If $\left\{x_{n}^{\prime}\right\}_{n=1}^{\infty}$ is an equicontinuous, pairwise disjoint sequence in $E^{\prime}$, then $x_{n}^{\prime} \rightarrow 0$ with respect to $\sigma\left(E^{\prime}, E\right)$.

Proof. Since $\left\{x_{n}^{\prime}\right\}_{n=1}^{\infty}$ is equicontinuous, there exists a continuous Riesz seminorm $r$ on $E$ such that $\left|\left\langle x, x_{n}^{\prime}\right\rangle\right| \leq r(x)$ for all $x \in E$ and $n \in \mathbb{N}$. Since $\left\langle|x|,\left|x_{n}^{\prime}\right|\right\rangle=\sup \left\{\left|\left\langle y, x_{n}^{\prime}\right\rangle\right|:|y| \leq|x|\right\}$, the disjoint sequence $\left\{\left|x_{n}^{\prime}\right|\right\}_{n=1}^{\infty}$ is also equicontinuous. Therefore, we may assume, without loss of generality, that $x_{n}^{\prime} \geq 0$ for all $n$.

Suppose that $x_{n}^{\prime} \rightarrow 0$ relative to $\sigma\left(E^{\prime}, E\right)$, i.e., there exists $x \in E$ such that $\left\langle x, x_{n}^{\prime}\right\rangle \nrightarrow 0$ as $n \rightarrow \infty$. Since $\left|\left\langle x, x_{n}^{\prime}\right\rangle\right| \leq\left\langle|x|, x_{n}^{\prime}\right\rangle$, we may assume that $x \in E^{+}$. By passing to a subsequence, if necessary, there exists $\delta>0$ such that $\left\langle x, x_{n}^{\prime}\right\rangle \geq \delta$ for all $n \in \mathbb{N}$. Since $\sup _{n \in \mathbb{N}}\left\langle x, x_{n}^{\prime}\right\rangle \leq r(x)<\infty$, it follows from [24], Theorem 2.3.1 (applied in the principal ideal $E_{x}$ ) that, by passing to a subsequence if 
necessary, there exist a pairwise disjoint sequence $\left\{v_{n}\right\}_{n=1}^{\infty}$ in $[0, x]$ and $\varepsilon>0$ such that $\left\langle v_{n}, x_{n}^{\prime}\right\rangle \geq \varepsilon$ for all $n$. The topology in $E$ is pre-Lebesgue and so $v_{n} \rightarrow 0$ in $E$ (see [4], Theorem 10.1). In particular, $r\left(v_{n}\right) \rightarrow 0$ as $n \rightarrow \infty$. Since $\left\langle v_{n}, x_{n}^{\prime}\right\rangle \leq r\left(v_{n}\right), n \in \mathbb{N}$, this yields a contradiction. Therefore, we may conclude that $\left\{x_{n}^{\prime}\right\}_{n=1}^{\infty}$ is a null sequence relative to $\sigma\left(E^{\prime}, E\right)$.

Remark 2.8 Let $E$ be a lc-solid Riesz space and suppose that $F \subseteq E$ is a lattice copy of $c_{0}$. Let $J: c_{0} \rightarrow F$ be a Riesz homeomorphism. For every continuous Riesz seminorm $p$ on $E$ there exists a constant $C_{p} \geq 0$ such that $p(J \lambda) \leq C_{p}\|\lambda\|_{\infty}$ for $\lambda \in c_{0}$. There also exists a continuous Riesz seminorm $q$ on $E$ such that $\|\lambda\|_{\infty} \leq q(J \lambda)$ for $\lambda \in c_{0}$. For each $n \in \mathbb{N}$, define the positive linear functional $\varphi_{n}$ on $F$ by $\left\langle x, \varphi_{n}\right\rangle=\left(J^{-1} x\right)(n), x \in F$. Since

$$
\left|\left\langle x, \varphi_{n}\right\rangle\right|=\left|\left(J^{-1} x\right)(n)\right| \leq\left\|J^{-1} x\right\|_{\infty} \leq q(x), \quad x \in F,
$$

it follows from Theorem 2.1 that, for each $n \in \mathbb{N}$, there exists a positive linear functional $\psi_{n}$ on $E$ such that $\left.\psi_{n}\right|_{F}=\varphi_{n}$ and $\left|\left\langle x, \psi_{n}\right\rangle\right| \leq q(x)$ for $x \in E$ (and so, $\left.0 \leq \psi_{n} \in E^{\prime}\right)$. If it is possible to choose the functionals $\psi_{n}(n \in \mathbb{N})$ such that $\left\langle x, \psi_{n}\right\rangle \rightarrow 0$ as $n \rightarrow \infty$ for all $x \in E$, then $F$ is positively complemented in $E$. Indeed, if this is the case, then the linear map $P: E \rightarrow E$ defined by

$$
P x=J\left(\left(\left\langle x, \psi_{n}\right\rangle\right)\right), \quad x \in E,
$$

is easily verified to be a positive continuous projection onto $F$.

Corollary 2.9 If $E$ is a lc-solid Riesz space with pre-Lebesgue topology, then any lattice copy of $c_{0}$ in $E$ is positively complemented.

Proof. Suppose that $F$ is a Riesz subspace of $E$ for which there exists a Riesz homeomorphism $J: c_{0} \rightarrow F$. Let the vectors $e_{n} \in F(n \in \mathbb{N})$ correspond to the unit basis vectors in $c_{0}$. Using the notation introduced in Remark 2.8, let $0 \leq x_{n}^{\prime} \in E^{\prime}$ be the minimal positive extension of the restriction of $\left.\psi_{n}\right|_{E_{e_{n}}}$. The sequence $\left\{x_{n}^{\prime}\right\}_{n=1}^{\infty}$ is pairwise disjoint and $\left.x_{n}^{\prime}\right|_{F}=\varphi_{n}$ for all $n$ (cf. the proof of Proposition 2.2 (i)). Since $\left|\left\langle x, x_{n}^{\prime}\right\rangle\right| \leq\left\langle|x|, x_{n}^{\prime}\right\rangle \leq\left\langle|x|, \psi_{n}\right\rangle \leq q(x)$ for all $x \in E$ and $n \in \mathbb{N}$, it follows that $\left\{x_{n}^{\prime}\right\}_{n=1}^{\infty}$ is equicontinuous. Hence, Lemma 2.7 applied to the sequence $\left\{x_{n}^{\prime}\right\}_{n=1}^{\infty}$ implies that $\left(\left\langle x, x_{n}^{\prime}\right\rangle\right) \in c_{0}$ for all $x \in E$. As observed in Remark 2.8, this implies that the map $P: E \rightarrow E$ given by $P x=J\left(\left(\left\langle x, x_{n}^{\prime}\right\rangle\right)\right), x \in E$, is a linear positive continuous projection onto $F$.

\section{Proofs of Theorems 1.1 and 1.2}

In this section we will present the proofs of Theorems 1.1 and 1.2. Actually, we will prove the results in a more general setting (so that these results also apply to the duals of Fréchet lattices).

For lc-solid Riesz spaces the following characterization of semireflexivity is relevant (see [4], Theorem 22.4). It should be recalled that a lc-solid Riesz space $E$ has a Levi topology (or, has the Levi property) if every upwards directed, topologically bounded system in $E^{+}$has a supremum in $E^{+}([4]$, p.61). In this case, $E$ is necessarily Dedekind complete. 
Proposition 3.1 If $E$ is a lc-solid Riesz space, then $E$ is semireflexive if and only if the topology in $E$ is both Lebesgue and Levi and the topology in $E_{\beta}^{\prime}$ is Lebesgue.

Theorem 1.2 is a special case of the following result.

Proposition 3.2 Suppose that $E$ is a lc-solid Riesz space such that:

(a) E is Dedekind $\sigma$-complete;

(b) $E$ is topologically complete;

(c) countable bounded subsets of $E_{\beta}^{\prime}$ are equicontinuous.

The following statements are equivalent.

(i) $E$ is not semireflexive.

(ii) E contains a positively complemented lattice copy of $\ell_{\infty}$, or $c_{0}$, or $\ell_{1}$.

Proof. Only (i) $\Rightarrow$ (ii) requires a proof. Assuming that $E$ is not semireflexive, Proposition 3.1 yields three possibilities:

(I) the topology of $E$ is not Lebesgue;

(II) the topology of $E$ is Lebesgue but, not Levi;

(III) the topology of $E_{\beta}^{\prime}$ is not Lebesgue.

In case (I) it follows from [4], Theorem 10.3, that the topology of $E$ is not pre-Lebesgue. Since $E$ is Dedekind $\sigma$-complete, it follows from [4], Theorem 10.7 , that $E$ contains a lattice copy of $\ell_{\infty}$, which is positively complemented by Remark 2.3 (b).

In case (II) it follows from [27], Theorem 1, that $E$ contains a lattice copy of $c_{0}$. Since the topology in $E$ is Lebesgue (and hence, pre-Lebesgue; see [4], Theorem 10.3), it follows from Corollary 2.9 that this copy of $c_{0}$ is positively complemented.

Finally, consider case (III). Since order intervals in $E_{\beta}^{\prime}$ are always topologically complete ([4], Theorem 19.13), it follows that $E_{\beta}^{\prime}$ does not have the pre-Lebesgue property (an inspection of the proof of [4], Theorem 10.3, shows that topological completeness of order intervals suffices). Since $E_{\beta}^{\prime}$ is Dedekind complete, it follows from [4], Theorem 10.7, that $E_{\beta}^{\prime}$ contains a lattice copy of $\ell_{\infty}$ and hence, a lattice copy of $c_{0}$. Proposition 2.4 now implies that $E$ contains a positively complemented lattice copy of $\ell_{1}$. The proof is complete.

Observe that, for cases (I) and (II) in the proof of Proposition 3.2, condition (c) on the space $E$ is not required.

Before proving our next result, the following observations will be useful. A lcHs $E$ is semireflexive if and only if every bounded subset of $E$ is relatively $\sigma\left(E, E^{\prime}\right)$-compact (see [22], Proposition 23.18). In particular, if $E$ is semireflexive, then every bounded sequence $\left\{x_{n}\right\}_{n=1}^{\infty}$ in $E$ has a $\sigma\left(E, E^{\prime}\right)$-cluster point (that is, there exists $y \in E$ such that every $\sigma\left(E, E^{\prime}\right)$-neighbourhood of $y$ contains $x_{n}$ for infinitely many values of $\left.n\right)$. If $T \in \mathcal{L}(E)$ is power bounded and $x \in E$, then it follows via an argument analogous to the one used in 
the proof of [20], Chapter 2, Theorem 1.1 (replacing the norm by seminorms), that $\lim _{n \rightarrow \infty} T_{[n]} x$ exists in $E$ if and only if the sequence $\left\{T_{[n]} x\right\}_{n=1}^{\infty}$ has a $\sigma\left(E, E^{\prime}\right)$-cluster point in $E$ (where $T_{[n]}$ is defined by (5)). Since $\left\{T^{n}\right\}_{n=1}^{\infty}$ is equicontinuous, for each $x \in E$ the set $\left\{T_{[n]} x: n \in \mathbb{N}\right\}$ is bounded in $E$. Consequently, if $E$ is semireflexive, then for all $x \in E$ the sequence $\left\{T_{[n]} x\right\}_{n=1}^{\infty}$ has a $\sigma\left(E, E^{\prime}\right)$-cluster point in $E$ and so, $\lim _{n \rightarrow \infty} T_{[n]} x$ exists in $E$. This establishes the following result.

Proposition 3.3 Every semireflexive lcHs is mean ergodic.

Remark 3.4 (a) Proposition 3.3 improves Proposition 2.3 of [2], where the assumptions on the lcHs $E$ are that it should be reflexive and have the property that relatively $\sigma\left(E, E^{\prime}\right)$-compact sets are relatively sequentially $\sigma\left(E, E^{\prime}\right)$-compact (in which case $E$ is mean ergodic). Accordingly, several other results in [2], namely Proposition 2.4 and Theorems 3.5 and 3.7, can also be extended by removing the requirement that "relatively $\sigma\left(E, E^{\prime}\right)$ compact sets are relatively sequentially $\sigma\left(E, E^{\prime}\right)$-compact" and replacing the use of Proposition 2.3 of [2] in their proofs with Proposition 3.3 above.

(b) Suppose that $E$ is a lcHs and $F$ is a closed complemented subspace of $E$ (that is, $F$ is the range of a continuous projection $P$ in $E)$. Let $T \in \mathcal{L}(F)$. Considering $T P$ as a continuous operator from $E$ into itself, it is clear that $(T P)^{n}=T^{n} P$ and $(T P)_{[n]}=T_{[n]} P$ for all $n \in \mathbb{N}$. Evidently, the operator $T P$ is power bounded in $E$ whenever $T$ is power bounded in $F$. Moreover, if $T$ is not mean ergodic in $F$, then $T P$ is not mean ergodic in $E$. Note that, if $E$ is a lc-solid Riesz space and $F$ is a Riesz subspace, then TP is positive whenever both $T$ and $P$ are positive.

Proposition 3.5 Let $E$ be a lc-solid Riesz space satisfying conditions (a), (b) and (c) of Proposition 3.2. The following statements are equivalent.

(i) E is semireflexive.

(ii) E is mean ergodic.

(iii) Every positive power bounded linear operator in $E$ is mean ergodic.

Proof. Implication (i) $\Rightarrow$ (ii) is Proposition 3.3 and (ii) $\Rightarrow$ (iii) is trivial. To show that (iii) $\Rightarrow$ (i), suppose that $E$ is not semireflexive. It follows from Proposition 3.2 that $E$ contains a positively complemented lattice copy $F$ of $\ell_{\infty}$, or $c_{0}$, or $\ell_{1}$. As observed before (see (4)), there then exists a positive power bounded linear operator $T$ in $F$ which is not mean ergodic. Via Remark 3.4 (b), this implies that $E$ does not satisfy statement (iii). The proof is complete.

To treat the case where the space $E$ is not Dedekind $\sigma$-complete, the following result will be needed. In [1], Theorem 1.6, it is shown that if a Fréchet space $E$ contains a copy of the Banach space $c_{0}$, then $E$ is not mean ergodic. The same conclusion holds in any sequentially complete lcHs $E$; see [2], Theorem 3.8. The next proposition exhibits a similar result for lc-solid Riesz spaces, without any sequential completeness requirement. The proof of this result uses some ideas from the proof of [14], Proposition 1. 
Proposition 3.6 Suppose that $E$ is a lc-solid Riesz space. If $E$ contains a lattice copy of $c_{0}$, then there exists a regular power bounded operator on $E$ which is not mean ergodic. In particular, $E$ is not mean ergodic.

Proof. Suppose that $F$ is a lattice copy of $c_{0}$ in $E$ and let $J: c_{0} \rightarrow F$ be a Riesz homeomorphism. Let the vectors $\left\{u_{n}\right\}_{n=1}^{\infty} \subseteq F$ correspond to the unit basis vectors in $c_{0}$, so that

$$
J(\lambda)=\sum_{n=1}^{\infty} \lambda_{n} u_{n}, \quad \lambda=\left(\lambda_{n}\right) \in c_{0}
$$

Since $J$ is a linear homeomorphism, there exists a continuous Riesz seminorm $q$ on $E$ such that

$$
\|\lambda\|_{\infty} \leq q(J \lambda), \quad \lambda \in c_{0}
$$

Moreover, for every continuous Riesz seminorm $p$ on $E$ there exists a constant $C_{p} \geq 0$ such that

$$
p(J \lambda) \leq C_{p}\|\lambda\|_{\infty}, \quad \lambda \in c_{0}
$$

For $n \in \mathbb{N}$, define the linear functional $0 \leq \varphi_{n} \in F^{\prime}$ by

$$
\left\langle x, \varphi_{n}\right\rangle=\left(J^{-1} x\right)(n), \quad x \in F .
$$

It follows from (13) that

$$
\left|\left\langle x, \varphi_{n}\right\rangle\right| \leq\left\|J^{-1} x\right\|_{\infty} \leq q(x), \quad x \in F,
$$

and so Theorem 2.1 implies that there exists $0 \leq \psi_{n} \in E^{\sim}$ such that $\left.\psi_{n}\right|_{F}=\varphi_{n}$ and $\left|\left\langle x, \psi_{n}\right\rangle\right| \leq q(x)$ for $x \in E$ (so, in particular, $\psi_{n} \in E^{\prime}$ ). If $\left\langle x, \psi_{n}\right\rangle \rightarrow 0$ as $n \rightarrow \infty$ for all $x \in E$, then $F$ is positively complemented in $E$ (see Remark 2.8), in which case it follows that there exists a positive power bounded operator on $E$ which is not mean ergodic (see the proof of Proposition 3.5). In this case we are done.

So, assume that there exists $0<u \in E$ such that $\left\langle u, \psi_{n}\right\rangle \nrightarrow 0$ as $n \rightarrow \infty$. Fix a sequence $\left\{\alpha_{n}\right\}_{n=1}^{\infty}$ in $\mathbb{R}$ satisfying $0<\alpha_{n}<1$ for all $n$ with $\alpha_{n} \rightarrow 0$ as $n \rightarrow \infty$. Define the positive linear operator $B_{1}: E \rightarrow E$ by

$$
B_{1} x=J\left(\left(\alpha_{n}\left\langle x, \psi_{n}\right\rangle\right)\right)=\sum_{n=1}^{\infty} \alpha_{n}\left\langle x, \psi_{n}\right\rangle u_{n}, \quad x \in E
$$

Note that $B_{1}$ is well defined as $\left|\left\langle x, \psi_{n}\right\rangle\right| \leq q(x)$ for all $n \in \mathbb{N}$ implies that $\left(\alpha_{n}\left\langle x, \psi_{n}\right\rangle\right) \in c_{0}$ for all $x \in E$. If $p$ is any continuous Riesz seminorm on $E$, then it follows from (14) that

$$
p\left(B_{1} x\right) \leq C_{p}\left\|\left(\alpha_{n}\left\langle x, \psi_{n}\right\rangle\right)\right\|_{\infty} \leq C_{p}\left\|\left(\left\langle x, \psi_{n}\right\rangle\right)\right\|_{\infty} \leq C_{p} q(x), \quad x \in E,
$$

and so $B_{1}$ is continuous. Define the regular operator $T \in \mathcal{L}(E)$ by $T=I-B_{1}$. Using (15) and the identities $\left\langle u_{n}, \psi_{k}\right\rangle=\delta_{k n}$ for all $k, n \in \mathbb{N}$, it follows that $T^{k}=I-B_{k}$, where

$$
B_{k} x=\sum_{n=1}^{\infty} \beta_{n}^{(k)}\left\langle x, \psi_{n}\right\rangle u_{n}, \quad x \in E
$$


and $\beta_{n}^{(k)}=1-\left(1-\alpha_{n}\right)^{k}$ for all $k, n \in \mathbb{N}$. Observe that $0 \leq \beta_{n}^{(k)} \uparrow_{k} 1$ for all $n$ and that

$$
0 \leq B_{1} \leq B_{2} \leq \cdots .
$$

Furthermore, if $p$ is any continuous Riesz seminorm on $E$, then

$$
\begin{aligned}
p\left(T^{k} x\right) & \leq p(x)+p\left(B_{k} x\right) \leq p(x)+C_{p}\left\|\left(\beta_{n}^{(k)}\left\langle x, \psi_{n}\right\rangle\right)\right\|_{\infty} \\
& \leq p(x)+C_{p}\left\|\left(\left\langle x, \psi_{n}\right\rangle\right)\right\|_{\infty} \leq p(x)+C_{p} q(x)
\end{aligned}
$$

for all $x \in E$ and $k \in \mathbb{N}$. Hence, the operator $T$ is power bounded. Defining $S_{n}=n^{-1}\left(B_{1}+\cdots+B_{n}\right)$, it is clear that

$$
T_{[n]}=I-S_{n}, \quad n \in \mathbb{N},
$$

and $0 \leq S_{1} \leq S_{2} \leq \cdots$. Hence, if $x \in E$, then $\lim _{n \rightarrow \infty} T_{[n]} x$ exists if and only if $\lim _{n \rightarrow \infty} S_{n} x$ exists.

Suppose that $0 \leq x \in E$ is such that $y=\lim _{n \rightarrow \infty} S_{n} x$ exists (in which case $S_{n} x \uparrow_{n} y$; [4], Theorem 5.6 (iii)). Using (17) and the fact that any increasing Cesàro convergent sequence is itself convergent, it follows that $B_{n} x \rightarrow y$ as $n \rightarrow \infty$ (and so $\left.0 \leq B_{n} x \uparrow_{n} y\right)$. Observe that

$$
\begin{aligned}
y & =\bigvee_{k=1}^{\infty} B_{k} x=\bigvee_{k=1}^{\infty} \bigvee_{n=1}^{\infty} \beta_{n}^{(k)}\left\langle x, \psi_{n}\right\rangle u_{n} \\
& =\bigvee_{n=1}^{\infty} \bigvee_{k=1}^{\infty} \beta_{n}^{(k)}\left\langle x, \psi_{n}\right\rangle u_{n}=\bigvee_{n=1}^{\infty}\left\langle x, \psi_{n}\right\rangle u_{n} .
\end{aligned}
$$

On the other hand, $B_{n} x \in F^{+}$for all $n$ and so $y \in F^{+}$. Hence, there exists $\lambda=\left(\lambda_{n}\right) \in c_{0}^{+}$such that $y=J \lambda=\bigvee_{n=1}^{\infty} \lambda_{n} u_{n}$ and so, $\bigvee_{n=1}^{\infty}\left\langle x, \psi_{n}\right\rangle u_{n}=$ $\bigvee_{n=1}^{\infty} \lambda_{n} u_{n}$. Since $\left\{u_{n}\right\}_{n=1}^{\infty}$ is a disjoint sequence, it follows that $\left\langle x, \psi_{n}\right\rangle=\lambda_{n}$ for all $n$ and hence, $\left(\left\langle x, \psi_{n}\right\rangle\right) \in c_{0}$.

We thus have shown that if $0 \leq x \in E$ is such that $\lim _{n \rightarrow \infty} T_{[n]} x$ exists, then $\left(\left\langle x, \psi_{n}\right\rangle\right) \in c_{0}$. Since we assumed that there exists $0<u \in E$ such that $\left\langle u, \psi_{n}\right\rangle \nrightarrow 0$ as $n \rightarrow \infty$, this shows that the regular power bounded operator $T$ is not mean ergodic. The proof is complete.

As observed in [27], Lemma 1, any sequentially complete lc-solid Riesz space which is not Dedekind $\sigma$-complete contains a lattice copy of $c_{0}$. Together with Proposition 3.6, this yields the following result.

Corollary 3.7 If E is a sequentially complete lc-solid Riesz space which is mean ergodic, then $E$ is Dedekind $\sigma$-complete.

In combination with Propositions 3.3 and 3.5 we obtain the following consequence.

Corollary 3.8 If E is a topologically complete lc-solid Riesz space such that countable bounded subsets of $E_{\beta}^{\prime}$ are equicontinuous, then the following statements are equivalent.

(i) E is semireflexive.

(ii) E is mean ergodic. 
(iii) $E$ is Dedekind $\sigma$-complete and every positive power bounded linear operator in $E$ is mean ergodic.

The proof of Theorem 1.1 is now a simple consequence of the previous results. Proof. (of Theorem 1.1) Let $E$ be a Fréchet lattice. The equivalence of statements (i), (ii) and (iii) is a special case of Corollary 3.8. The strong dual $E_{\beta}^{\prime}$ of $E$ is a topologically complete and Dedekind complete lc-solid Riesz space for which countable bounded subsets of $\left(E_{\beta}^{\prime}\right)_{\beta}^{\prime}$ are equicontinuous (see the discussion prior to Theorem 2.1 and Remark 2.5 (i)). Consequently, Proposition 3.5 may be applied to $E_{\beta}^{\prime}$ and so (iv) holds if and only if $E_{\beta}^{\prime}$ is semireflexive.

If $E$ is reflexive, then $E_{\beta}^{\prime}$ is reflexive (see [22], Corollary 25.11) and hence, semireflexive. Assume now that $E_{\beta}^{\prime}$ is semireflexive. Since $E$ is a Fréchet space, its topology coincides with the Mackey topology ([19], p. 261, (4)). Since $E$ is complete, it follows from [19], p. 303, (6), that $E$ is reflexive. This shows that statements (iv) and (i) in Theorem 1.1 are equivalent. The proof is complete.

Remark 3.9 Let $E$ be a topologically complete lc-solid Riesz space. If $E$ is mean ergodic, then it follows from Corollary 3.7 and the proof of Proposition 3.2 that $E$ has both the Lebesgue and the Levi property. In particular, $E$ is Dedekind complete.

\section{Proof of Theorem 1.3}

Recall that a linear map $T$ on a Riesz space $E$ is called power order bounded if for every $x \in E^{+}$there exists $z \in E^{+}$such that

$$
\bigcup_{n=0}^{\infty} T^{n}([-x, x]) \subseteq[-z, z] .
$$

Note that (18) is equivalent to saying that $\left|T^{n} y\right| \leq z$ for all $n=0,1, \ldots$ whenever $|y| \leq x$.

Proposition 4.1 If $E$ is a complete barrelled lc-solid Riesz space, then the following statements are equivalent.

(i) The topology of E is Lebesgue.

(ii) Every power order bounded operator on $E$ is mean ergodic.

Proof. (i) $\Rightarrow$ (ii). Let $T$ be a power order bounded operator in $E$. As observed in Section 1, this implies that $T$ is power bounded (as $E$ is assumed to be barrelled). Given $x \in E$, let $z \in E^{+}$satisfy (18) for $|x|$, which implies, in particular, that $\left|T^{n} x\right| \leq z$ for all $n \in \mathbb{N}$. Consequently, $\left|T_{[n]} x\right| \leq z$ for all $n \in \mathbb{N}$, that is, the sequence $\left\{T_{[n]} x\right\}_{n=1}^{\infty}$ is contained in the order interval $[-z, z]$. Since $E$ is topologically complete and its topology is Lebesgue, it follows that $E$ is Dedekind complete (see [4], Theorem 10.3). Hence, by [4], Theorem 22.1, the order interval $[-z, z]$ is $\sigma\left(E, E^{\prime}\right)$-compact and so, the set $\left\{T_{[n]} x: n \in \mathbb{N}\right\}$ is relatively $\sigma\left(E, E^{\prime}\right)$-compact. Therefore, the sequence $\left\{T_{[n]} x\right\}_{n=1}^{\infty}$ has a $\sigma\left(E, E^{\prime}\right)$ cluster point in $E$, which implies that $\lim _{n \rightarrow \infty} T_{[n]} x$ exists in $\bar{E}$ (see the discussion prior to Proposition 3.3). Hence, $T$ is mean ergodic. 
(ii) $\Rightarrow$ (i). Suppose that the topology in $E$ is not Lebesgue. Since $E$ is complete it follows from [4], Theorem 10.3, that the topology is not pre-Lebesgue. Therefore, there exist $0<u \in E$ and a disjoint sequence $\left\{u_{n}\right\}_{n=1}^{\infty}$ in $[0, u]$ such that $u_{n} \nrightarrow 0$ as $n \rightarrow \infty$ ([4], Theorem 10.1). Hence, there exists a continuous Riesz seminorm $r$ on $E$ such that $r\left(u_{n}\right) \nrightarrow 0$ as $n \rightarrow \infty$. By passing to a subsequence, if necessary, we may assume that $r\left(u_{n}\right) \geq \delta$ for all $n$ and some $\delta>0$. Define the injective Riesz homomorphism $J_{0}: c_{00} \rightarrow E$ by

$$
J_{0} \lambda=\sum_{k=1}^{n} \lambda_{k} u_{k}, \quad \lambda=\left(\lambda_{1}, \ldots, \lambda_{n}, 0,0, \ldots\right) \in c_{00} .
$$

Since $\left|J_{0} \lambda\right| \leq\|\lambda\|_{\infty} u$, it follows that $p\left(J_{0} \lambda\right) \leq p(u)\|\lambda\|_{\infty}, \lambda \in c_{00}$, for all continuous Riesz seminorms $p$ on $E$. The inequalities $\left|J_{0} \lambda\right| \geq\left|\lambda_{k}\right| u_{k}$ for all $k$, imply that $r\left(J_{0} \lambda\right) \geq \delta\|\lambda\|_{\infty}$. Since $E$ is complete, it follows that $J_{0}$ extends continuously to a Riesz homeomorphism $J$ from $c_{0}$ onto a closed Riesz subspace $F$ of $E$ satisfying $p(J \lambda) \leq p(u)\|\lambda\|_{\infty}, \lambda \in c_{0}$, for all continuous Riesz seminorms $p$ on $E$. Moreover, $\|\lambda\|_{\infty} \leq q(J \lambda)$ for $\lambda \in c_{0}$, where $q=\delta^{-1} r$. Consequently, we are in the situation of Proposition 3.6. Let $\left\{\psi_{n}\right\}_{n=1}^{\infty}$ be the sequence in $\left(E^{\prime}\right)^{+}$as defined in the proof of Proposition 3.6 and observe that $\left\langle u, \psi_{n}\right\rangle \geq\left\langle u_{n}, \psi_{n}\right\rangle=1$ for all $n$. Therefore, if we define $T=I-B_{1}$, where the positive linear operator $B_{1}$ is given by (15), then $T$ is power bounded but not mean ergodic (see the proof of Proposition 3.6).

We claim that $T$ is power order bounded. Recall that $T^{k}=I-B_{k}, k \in \mathbb{N}$, where the positive operators $B_{k}$ are given by (16). Let $x \in E^{+}$and $y \in E$ satisfy $|y| \leq x$. Using $0 \leq\left\langle x, \psi_{n}\right\rangle \leq q(x)$ for all $n$, it follows easily that

$$
\left|T^{k} y\right| \leq|y|+\left|B_{k} y\right| \leq x+B_{k} x \leq x+q(x) u, \quad k \in \mathbb{N}
$$

which establishes the claim. We thus have shown that if the topology of $E$ is not Lebesgue, then there exists a power order bounded operator on $E$ which is not mean ergodic. The proof is complete.

Since Fréchet lattices are complete barrelled lc-solid Riesz spaces, Theorem 1.3 is a special case of Proposition 4.1.

\section{Uniform mean ergodicity}

Recall that a power bounded linear operator $T \in \mathcal{L}(E)$, with $E$ a lcHs, is called uniformly mean ergodic if the Cesàro means $T_{[n]}, n \in \mathbb{N}$, (as defined by (5)) are convergent in $\mathcal{L}(E)$ with respect to the uniform operator topology $\tau_{b}$ (defined via the seminorms $q_{B}$ in $\mathcal{L}(E)$ given by $\left.(3)\right)$. If $E$ is a lc-solid Riesz space, then the topology $\tau_{b}$ is generated by the seminorms $q_{B}$, where $q$ is a continuous Riesz seminorm on $E$ and $B \in \mathcal{B}_{s}$.

In the sequel, we denote by $Z(E)$ the centre of a lc-solid Riesz space $E$ (see [29], Chapter 20, or [24], Section 3.1). The Boolean algebra $\mathcal{P}(E)$ of all band projections in $E$ (cf. [21], Section 30) coincides with the Boolean algebra of all idempotents in $Z(E)$ (equivalently, the Boolean algebra of all components of the identity operator $I$ in $Z(E))$. If $T \in Z(E)$, then, by definition, there exists $0 \leq \lambda \in \mathbb{R}$ such that $|T x| \leq \lambda|x|, x \in E$, and so $Z(E) \subseteq \mathcal{L}(E)$. Furthermore, $Z(E)$ is a commutative subalgebra of $\mathcal{L}(E)$ and $Z(E)$ is an $f$-algebra (see e.g. 
[24], Section 3.1 or [29], Section 140, for the definition). If $q$ is a continuous Riesz seminorm on $E$ and $B \in \mathcal{B}_{s}$, then $q_{B}$ is a Riesz seminorm on $Z(E)$. Indeed, if $|S| \leq|T|$ in $Z(E)$, then $|S x|=|S||x| \leq|T||x|=|T x|$ and hence, $q(S x) \leq q(T x)$ for all $x \in E$, which implies that $q_{B}(S) \leq q_{B}(T)$. Consequently, equipped with the topology $\tau_{b}, Z(E)$ is a lc-solid Riesz space. It should also be observed that an operator $T \in Z(E)$ is power bounded if and only if $|T| \leq I$.

Theorem 5.1 If E is a Dedekind $\sigma$-complete lc-solid Riesz space in which order intervals are topologically complete, then the following statements are equivalent.

(i) Every power bounded $T \in Z(E)$ is uniformly mean ergodic.

(ii) Every topologically bounded, disjoint sequence in E converges to zero.

(iii) Every disjoint sequence of band projections in $E$ converges to zero with respect to $\tau_{b}$.

(iv) $\mathcal{P}(E)$ is a $\tau_{b}$-Bade complete Boolean algebra of projections, that is, $\mathcal{P}(E)$ is a complete Boolean algebra and $P_{\alpha} \uparrow_{\alpha} P$ in $\mathcal{P}(E)$ implies that $P_{\alpha} \rightarrow_{\alpha} P$ with respect to $\tau_{b}$.

Proof. (i) $\Rightarrow$ (ii). Suppose that $\left\{u_{n}\right\}_{n=1}^{\infty}$ is a topologically bounded, disjoint sequence in $E^{+}$. Let $P_{n}$ denote the band projection in $E$ onto the principal band $\left\{u_{n}\right\}^{d d}$ generated by $u_{n}$ (recall that a Dedekind $\sigma$-complete Riesz space has the principal projection property; see [21], Section 25) and observe that $P_{m} P_{n}=0$ whenever $m \neq n$. Fix a sequence $\left\{\alpha_{n}\right\}_{n=1}^{\infty}$ in $\mathbb{R}$ satisfying $0<\alpha_{n}<1$ for all $n$ and $\alpha_{n} \uparrow_{n} 1$. If $x \in E^{+}$, then

$$
0 \leq \sum_{n=1}^{N} \alpha_{n} P_{n} x \uparrow_{N} \leq x
$$

and so, $T x=\sum_{n=1}^{\infty} \alpha_{n} P_{n} x=\sup _{N} \sum_{n=1}^{N} \alpha_{n} P_{n} x$ exists in $E$ (as $E$ is Dedekind $\sigma$-complete). Consequently,

$$
T x=\sum_{n=1}^{\infty} \alpha_{n} P_{n} x, \quad x \in E,
$$

exists as an order convergent series in $E$. Since $0 \leq T \leq I$, it is clear that $T \in Z(E) \subseteq \mathcal{L}(E)$ and $T$ is power bounded. It is easily verified that

$$
T^{k} x=\sum_{n=1}^{\infty} \alpha_{n}^{k} P_{n} x, \quad x \in E,
$$

for all $k \in \mathbb{N}$. Note that $0 \leq T^{k} \downarrow_{k}$. We claim that $T^{k} x \downarrow_{k} 0$ in $E$ for all $x \in E^{+}$. Indeed, suppose that $w \in E$ is such that $0 \leq w \leq T^{k} x$ for all $k \in \mathbb{N}$ and some $x \in E^{+}$. It follows from (19) that $0 \leq P_{n} w \leq \alpha_{n}^{k} P_{n} x$ for all $k, n \in \mathbb{N}$ and so, $P_{n} w=0$ for all $n$ (as $\left.\alpha_{n}^{k} \downarrow_{k} 0\right)$. This implies that $w \wedge P_{n} x=0$ for all $n$, so $w=w \wedge T x=0$, which proves the claim.

It is now easy to see that $0 \leq T_{[k]} x \downarrow_{k} 0$ for all $x \in E^{+}$. By hypothesis, there exists $S \in \mathcal{L}(E)$ such that $T_{[k]} \rightarrow S$ with respect to $\tau_{b}$ and so, in particular, $T_{[k]} x \rightarrow S x$ for all $x \in E^{+}$. Via [4], Theorem 5.6 (iii), it follows that $S x=0$ 
for all $x \in E^{+}$and hence, $S=0$. Consequently, $T_{[k]} \rightarrow 0$ with respect to $\tau_{b}$. Since $0 \leq T^{k} \leq T^{j}(1 \leq j \leq k)$, it follows that $0 \leq T^{k} \leq T_{[k]}$ and so, $q_{B}\left(T^{k}\right) \leq q_{B}\left(T_{[k]}\right)$ for every continuous Riesz seminorm $q$ and every $B \in \mathcal{B}_{s}$. Consequently, $T^{k} \rightarrow 0$ with respect to $\tau_{b}$. This implies, in particular, that $\lim _{k \rightarrow \infty} \sup _{n} p\left(T^{k} u_{n}\right)=0$ for every continuous Riesz seminorm $p$ on $E$. Since $T^{k} u_{n}=\alpha_{n}^{k} u_{n}$, it follows that $\lim _{k \rightarrow \infty} \sup _{n} \alpha_{n}^{k} p\left(u_{n}\right)=0$. Given $k \in \mathbb{N}$, there exists $N_{k} \in \mathbb{N}$ such that $\alpha_{n}^{k} \geq 1 / 2$ for all $n \geq N_{k}$ and so, $\sup _{n} \alpha_{n}^{k} p\left(u_{n}\right) \geq$ $2^{-1} \sup _{n>N_{k}} p\left(u_{n}\right)$. Therefore, $p\left(u_{n}\right) \rightarrow 0$ as $n \rightarrow \infty$, which shows that $u_{n} \rightarrow 0$ as $n \rightarrow \infty$ in $E$. If $\left\{x_{n}\right\}_{n=1}^{\infty} \subseteq E$ is any topologically bounded, disjoint sequence, then $\left\{\left|x_{n}\right|\right\}_{n=1}^{\infty}$ has the same properties and so $\left|x_{n}\right| \rightarrow 0$, which implies that $x_{n} \rightarrow 0$ as $n \rightarrow \infty$.

(ii) $\Rightarrow$ (iii). Let $\left\{P_{n}\right\}_{n=1}^{\infty}$ be a disjoint sequence in $\mathcal{P}(E)$ and suppose that $P_{n} \nrightarrow 0$ with respect to $\tau_{b}$. Then there exists a continuous Riesz seminorm $q$ on $E$ and $B \in \mathcal{B}_{s}$ such that $q_{B}\left(P_{n}\right) \nrightarrow 0$ as $n \rightarrow \infty$ (with $q_{B}$ given by (3)). By passing to a subsequence, if necessary, we may assume that $q_{B}\left(P_{n}\right) \geq \delta$ for all $n \in \mathbb{N}$ and some $\delta>0$. Hence, for each $n$ there exists $x_{n} \in B$ such that $q\left(P_{n} x_{n}\right) \geq \delta / 2$. Since $\left|P_{n} x_{n}\right|=P_{n}\left|x_{n}\right| \leq\left|x_{n}\right|$, the sequence $\left\{P_{n} x_{n}\right\}_{n=1}^{\infty}$ is bounded and disjoint and so, by hypothesis, $P_{n} x_{n} \rightarrow 0$ as $n \rightarrow \infty$. This contradicts the fact that $q\left(P_{n} x_{n}\right) \geq \delta / 2$ for all $n$. Hence, we may conclude that $P_{n} \rightarrow 0$ with respect to $\tau_{b}$.

(iii) $\Rightarrow$ (iv). First, observe that the topology in $E$ is pre-Lebesgue. Indeed, suppose that $x \in E^{+}$and that $\left\{x_{n}\right\}_{n=1}^{\infty}$ is a disjoint sequence in $[0, x]$. Denoting by $P_{n}$ the band projection in $E$ onto the principal band $\left\{x_{n}\right\}^{d d}$, it is clear that $\left\{P_{n}\right\}_{n=1}^{\infty}$ is a disjoint sequence in $\mathcal{P}(E)$ and so, by hypothesis, $P_{n} \rightarrow 0$ as $n \rightarrow \infty$ with respect to $\tau_{b}$. Since $0 \leq x_{n}=P_{n} x_{n} \leq P_{n} x$ for all $n$, it is now clear that $x_{n} \rightarrow 0$ as $n \rightarrow \infty$. Hence, the topology of $E$ is pre-Lebesgue. Since, by hypothesis, order intervals in $E$ are complete, this implies that $E$ has the Lebesgue property and $E$ is Dedekind complete (see [4], Theorem 10.3, and its proof, where it is only required that order intervals are complete). Consequently, $\mathcal{P}(E)$ is a complete Boolean algebra (see [21], Theorem 24.9 (i) and Theorem 30.6 (ii)).

We shall show next that $\tau_{b}$ is a pre-Lebesgue topology on $Z(E)$. Since $I$ is a strong order unit in $Z(E)$, it suffices to show that any disjoint sequence $\left\{T_{n}\right\}_{n=1}^{\infty}$ in $[0, I]$ converges to zero with respect to $\tau_{b}$. Denoting by $P_{n} \in \mathcal{P}(E)$ the component of $I$ in the principal band $\left\{T_{n}\right\}^{d d}$, it follows that $\left\{P_{n}\right\}_{n=1}^{\infty}$ is a disjoint sequence in $\mathcal{P}(E)$ satisfying $0 \leq T_{n}=T_{n} P_{n} \leq P_{n}$ for all $n$. By hypothesis, $P_{n} \rightarrow 0$ and so also $T_{n} \rightarrow 0$ as $n \rightarrow \infty$ with respect to $\tau_{b}$. Hence, $\tau_{b}$ is a pre-Lebesgue topology on $Z(E)$. Since order intervals in $E$ are complete, it is easily verified that order intervals in $Z(E)$ are complete with respect to $\tau_{b}$. Consequently, $\tau_{b}$ is a Lebesgue topology on $Z(E)$. In particular, if $P_{\alpha} \uparrow_{\alpha} P$ in $\mathcal{P}(E)$, then $P_{\alpha} \rightarrow_{\alpha} P$ with respect to $\tau_{b}$. We may conclude that $\mathcal{P}(E)$ is $\tau_{b}$-Bade complete.

(iv) $\Rightarrow$ (i). We start with the following simple observation. If $T \in Z(E)$ satisfies $-I \leq T \leq \alpha I$ for some $\alpha<1$, then $T_{[k]} \rightarrow 0$ as $k \rightarrow \infty$ with respect to $\tau_{b}$. Indeed, $I-T \geq(1-\alpha) I$ and so $(I-T)^{-1}$ exists in $Z(E)^{+}$(see [29], Theorem 146.3). This implies that

$$
T+T^{2}+\cdots+T^{k}=(I-T)^{-1}\left(T-T^{k+1}\right)
$$


and so,

$$
\left|T_{[k]}\right| \leq(1 / k)(I-T)^{-1}\left(|T|+|T|^{k+1}\right) \leq(2 / k)(I-T)^{-1}
$$

for all $k \in \mathbb{N}$. From this estimate it is clear that $T_{[k]} \rightarrow 0$ with respect to $\tau_{b}$.

Let $T \in Z(E)$ satisfy $|T| \leq I$ and fix $0<\alpha<1$. Let $P \in \mathcal{P}(E)$ be the component of $I$ in the band $\left\{(I-T)^{+}\right\}^{d}$, in which case $P T \leq P$. On the other hand, since $(P-P T)^{+}=P(I-T)^{+}=0$, it follows that $P \leq P T$ and hence, $P=P T$. This implies, in particular, that $P T_{[k]}=P$ for all $k \in \mathbb{N}$ (as $T$ and $P$ commute). Let $Q_{\alpha} \in \mathcal{P}(E)$ be the component of $I$ in the band $\left\{(\alpha I-T)^{+}\right\}^{d d}$. Note that $(\alpha I-T)^{+} \leq(I-T)^{+}$and so, $P Q_{\alpha}=0$. Since $\left(\alpha Q_{\alpha}-Q_{\alpha} T\right)^{-}=$ $Q_{\alpha}(\alpha I-T)^{-}=0$, it follows that $Q_{\alpha} T \leq \alpha Q_{\alpha}$ and so $-I \leq Q_{\alpha} T \leq \alpha I$. The previous paragraph implies that $Q_{\alpha} T_{[k]}=\left(Q_{\alpha} T\right)_{[k]} \rightarrow 0$ as $k \rightarrow \infty$ with respect to $\tau_{b}$. Writing

$$
T_{[k]}-P=(I-P) T_{[k]}=Q_{\alpha} T_{[k]}+\left(I-P-Q_{\alpha}\right) T_{[k]},
$$

it follows that

$$
\limsup _{k \rightarrow \infty} q_{B}\left(T_{[k]}-P\right) \leq \limsup _{k \rightarrow \infty} q_{B}\left(\left(I-P-Q_{\alpha}\right) T_{[k]}\right)
$$

whenever $q$ is a continuous Riesz seminorm on $E$ and $B \in \mathcal{B}_{s}$. Furthermore, $|T| \leq I$ yields $\left|T_{[k]}\right| \leq I$ and hence, $\left|\left(I-P-Q_{\alpha}\right) T_{[k]}\right| \leq I-P-Q_{\alpha}$. So, $q_{B}\left(\left(I-P-Q_{\alpha}\right) T_{[k]}\right) \leq q_{B}\left(I-P-Q_{\alpha}\right)$ for all $k$. Accordingly,

$$
\limsup _{k \rightarrow \infty} q_{B}\left(T_{[k]}-P\right) \leq q_{B}\left(I-P-Q_{\alpha}\right) .
$$

Now observe that $I-P-Q_{\alpha}$ is the component of $I$ in the band $\left\{(\alpha I-T)^{+}\right\}^{d} \cap$ $\left\{(I-T)^{+}\right\}^{d d}$. Furthermore, if $\alpha \uparrow 1$, then $\left\{(\alpha I-T)^{+}\right\}^{d d} \uparrow\left\{(I-T)^{+}\right\}^{d d}$ and so, $\left\{(\alpha I-T)^{+}\right\}^{d} \downarrow\left\{(I-T)^{+}\right\}^{d}$. Consequently, $I-P-Q_{\alpha} \downarrow 0$ as $\alpha \uparrow 1$. By the $\tau_{b}$-Bade completeness of $\mathcal{P}(E)$, this implies that $q_{B}\left(I-P-Q_{\alpha}\right) \downarrow 0$ as $\alpha \uparrow 1$ and so we may conclude that $\lim _{k \rightarrow \infty} q_{B}\left(T_{[k]}-P\right)=0$. This shows that $T_{[k]} \rightarrow P$ as $k \rightarrow \infty$ with respect to $\tau_{b}$. The proof is complete.

Remark 5.2 If $E$ is a lc-solid Riesz space, then $E_{\beta}^{\prime}$ is always Dedekind complete (see Section 2) and order intervals in $E_{\beta}^{\prime}$ are topologically complete ([4], Theorem 19.13). Consequently, Theorem 5.1 may always be applied in $E_{\beta}^{\prime}$.

An immediate consequence of the above theorem is the following result.

Corollary 5.3 If E is topologically complete lc-solid Riesz space which is uniformly mean ergodic, then every topologically bounded, disjoint sequence in $E$ converges to zero.

Proof. Since $E$ is, in particular, mean ergodic, it follows that $E$ is Dedekind complete (see Remark 3.9) and so, Theorem 5.1 applies. 
Theorem 1.4 follows immediately from Corollary 5.3. Indeed, if in a Banach lattice every norm bounded, disjoint sequence converges to zero, then every disjoint system in $E$ must be finite. This implies that $E$ is finite dimensional (see [21], Theorem 26.10).

It should be observed that any lcHs $E$ in which bounded sets are relatively compact is necessarily uniformly mean ergodic. Indeed, bounded subsets of $E$ are, in particular, relatively weakly compact and so, $E$ is semireflexive. This implies that $E$ is mean ergodic (see Proposition 3.3). Now, if $T \in \mathcal{L}(E)$ is power bounded, then the sequence $\left\{T_{[k]}\right\}_{k=1}^{\infty}$ is equicontinuous and convergent in $\mathcal{L}_{s}(E)$. Accordingly, the sequence $\left\{T_{[k]}\right\}_{k=1}^{\infty}$ also converges uniformly on all relatively compact subsets and hence, on all bounded subsets of $E$, that is, in $\mathcal{L}_{b}(E)$. Therefore, $E$ is uniformly mean ergodic.

If $E$ is a discrete and complete lc-solid Riesz space in which every bounded disjoint sequence converges to zero, then it follows from [4], Theorem 21.15, that every bounded set in $E$ is relatively compact. This observation, together with the previous paragraph, Corollary 5.3 and Theorem 5.1, yields the following result.

Corollary 5.4 If E is a topologically complete, discrete, lc-solid Riesz space, then the following statements are equivalent.

(i) $E$ is Dedekind $\sigma$-complete and every power bounded operator $T \in Z(E)$ is uniformly mean ergodic.

(ii) Every topologically bounded, disjoint sequence in E converges to zero.

(iii) Bounded subsets of $E$ are relatively compact.

(iv) E is uniformly mean ergodic.

It should be observed that a lc-solid Riesz space $E$ in which bounded sets are relatively compact, is necessarily discrete ([4], Corollary 21.13). Therefore, Theorem 1.5 is an immediate consequence of Corollary 5.4. The next example shows that the discreteness condition cannot be omitted in Corollary 5.4.

Example 5.5 Fix $1<p \leq \infty$ and define

$$
L^{p-}=\bigcap_{1 \leq q<p} L^{q}(0,1)
$$

where the interval $(0,1)$ is equipped with Lebesgue measure $\lambda$. Fixing a sequence $1<p_{1}<p_{2}<\cdots \uparrow p$, the Fréchet lattice topology in $L^{p-}$ is generated by the sequence $\left\{\|\cdot\|_{p_{k}}\right\}_{k=1}^{\infty}$ of Riesz norms. Moreover, $L^{p-}$ is reflexive. We claim that every bounded, disjoint sequence in $L^{p-}$ converges to zero. Indeed, let $\left\{u_{n}\right\}_{n=1}^{\infty}$ be a disjoint sequence in $L^{p-}$ such that $\sup _{n}\left\|u_{n}\right\|_{p_{k}}=C_{k}<\infty$ for all $k$. Defining $A_{n}=\left\{t \in(0,1):\left|u_{n}(t)\right|>0\right\}$, it is clear that $\left\{A_{n}\right\}_{n=1}^{\infty}$ consists of pairwise disjoint sets and so $\lambda\left(A_{n}\right) \rightarrow 0$ as $n \rightarrow \infty$. Given $k \in \mathbb{N}$, it follows from Hölder's inequality that

$$
\left\|u_{n}\right\|_{p_{k}} \leq\left\|u_{n}\right\|_{p_{k+1}} \lambda\left(A_{n}\right)^{\frac{1}{p_{k}}-\frac{1}{p_{k+1}}} \leq C_{k} \lambda\left(A_{n}\right)^{\frac{1}{p_{k}}-\frac{1}{p_{k+1}}}, \quad n \in \mathbb{N}
$$


Hence, $\left\|u_{n}\right\|_{p_{k}} \rightarrow 0$ as $n \rightarrow \infty$, which proves the claim. Consequently, the Dedekind complete Fréchet lattice $L^{p-}$ satisfies all (equivalent) statements of Theorem 5.1 (and so, in particular, statements (i) and (ii) of Corollary 5.4). It should be observed that the centre $Z\left(L^{p-}\right)$ may be identified with $L^{\infty}(0,1)$, acting on $L^{p-}$ via multiplication. Evidently, $L^{p-}$ is not discrete and hence (as is well known; [11]), it is not a Montel space (that is, $L^{p-}$ does not satisfy condition (ii) of Corollary 5.4). According to [1], Proposition 2.11, the space $L^{p-}$ is not uniformly mean ergodic. We point out that $L^{p-}$ cannot contain any closed Riesz subspace which is lattice isomorphic to an infinite dimensional Banach lattice $X$. For, all norm bounded, disjoint sequences in $X$ would converge to zero. As noted after Corollary 5.3, $X$ would then be finite dimensional. On the other hand, $L^{p-}$ does have a closed subspace which is topologically isomorphic to the Banach lattice $\ell^{2}$ ([1], Lemma 2.10).

It remains an interesting question whether every uniformly mean ergodic Fréchet lattice is actually discrete (and hence, Montel).

We end this paper with two observations concerning lc-solid Riesz spaces in which topologically bounded, disjoint sequences converge to zero.

Remark 5.6 Recall that a locally solid Riesz space $E$ is called (sequentially) monotone complete if every increasing Cauchy (sequence) net in $E$ is convergent.

(a) If $E$ is a monotone complete lc-solid Riesz space and all topologically bounded, disjoint sequences in $E$ converge to zero, then $E$ is semireflexive. Indeed, it follows from [4], Theorem 21.8, that all bounded subsets of $E$ are relatively weakly compact and hence, $E$ is semireflexive.

(b) If $E$ is a sequentially monotone complete lc-solid Riesz space, then the following two statements are equivalent.

(i) Every topologically bounded, disjoint sequence in $E$ converges to zero.

(ii) Every equicontinuous, disjoint sequence in $E_{\beta}^{\prime}$ converges to zero.

Indeed, this equivalence follows immediately from a result of Burkinshaw and Dodds ([4], Theorem 21.7, equivalence of (i) and (ii)). An inspection of the proof of [4], Theorem 21.7, shows that it actually suffices to assume that the space $E$ is sequentially monotone complete.

\section{References}

[1] A.A. Albanese, J. Bonet and W.J. Ricker, Mean ergodic operators in Fréchet spaces, Ann. Acad. Sci. Fenn. Math., 34 (2009), 401-436.

[2] A.A. Albanese, J. Bonet and W.J. Ricker, On mean ergodic operators; In: Vector Measures, Integration and Related Topics, Proc. of Conf. on "Vector Measures, Integration and Applications", Eichstätt, Sept. 2008, G.P. Curbera et. al. (Eds), Operator Theory: Advances and Applications 201, Birkhäuser Verlag, Basel, 2010, pp. 1-20. 
[3] A.A. Albanese, J. Bonet and W.J. Ricker, $C_{0}$-semigroups and mean ergodic operators in a class of Fréchet spaces, J. Math. Anal. Appl., 365 (2010), 142-157.

[4] C.D. Aliprantis and O. Burkinshaw, Locally Solid Riesz Spaces, Academic Press, New York-San Francisco-London, 1978.

[5] C.D. Aliprantis and O. Burkinshaw, Positive Operators, Academic Press, Orlando, 1985.

[6] M. Altman, Mean ergodic theorem in locally convex linear topological spaces, Studia Math. 13 (1953), 190-193.

[7] J. Bonet and M. Lindström, Spaces of operators between Fréchet spaces, Math. Proc. Camb. Phil. Soc. 115 (1994), 133-144.

[8] J. Bonet and W.J. Ricker, Mean ergodicity of multiplication operators in weighted spaces of holomorphic functions, Arch. Math. (Basel), 92 (2009), 428-437.

[9] R. del Campo and W.J. Ricker, The space of scalarly integrable functions for a Fréchet space valued measure, J. Math. Anal. Appl. 354 (2009), 641647.

[10] R. del Campo and W.J. Ricker, The Fatou completion of a Fréchet function space and applications, J. Aust. Math. Soc., to appear.

[11] J.M.F. Castillo, J.C. Diaz and J. Motos, On the Fréchet space $L^{p-}$, Manuscripta Math. 96 (1998), 219-230.

[12] J. Diestel, Sequences and Series in Banach Spaces, Springer-Verlag, BerlinHeidelberg-New York, 1984.

[13] N. Dunford and J.T. Schwartz, Linear Operators I: General Theory, 2nd ed., Wiley-Interscience, New York, 1964.

[14] E.Yu. Emel'yanov, Banach lattices on which every power bounded operator is mean ergodic, Positivity 1 (1997), 291-296.

[15] E.Yu. Emel'yanov and M.P.H. Wolff, Mean ergodicity on Banach lattices and Banach spaces, Arch. Math. (Basel) 72, (1999), 214-218.

[16] V.P. Fonf, M. Lin and P. Wojtaszczyk, Ergodic characterizations of reflexivity in Banach spaces, J. Funct. Anal. 187 (2001), 146-162.

[17] D.H. Fremlin, Topological Riesz Spaces and Measure Theory, Cambridge Univ. Press, Cambridge, 1974.

[18] E. Hille and R.S. Phillips, Functional Analysis and Semigroups, 4th printing of revised ed., Amer. Math. Soc., Providence, 1981.

[19] G. Köthe, Topological Vector Spaces I, 2nd rev. ed., Springer Verlag, BerlinHeidelberg-New York, 1983.

[20] U. Krengel, Ergodic Theorems, De Gruyter, Berlin, 1985. 
[21] W.A.J. Luxemburg and A.C. Zaanen, Riesz Spaces I, North Holland, Amsterdam, 1971.

[22] R.G. Meise and D. Vogt, Introduction to Functional Analysis, Clarendon Press, Oxford, 1997.

[23] G. Metafune and V.B. Moscatelli, On the spaces $\ell^{p+}=\bigcap_{q>p} \ell^{q}$, Math. Nachr. 147 (1990), 7-12.

[24] P. Meyer-Nieberg, Banach Lattices, Springer Verlag, Berlin-HeidelbergNew York, 1991.

[25] T.V. Panchapagesan, The Bartle-Dunford-Schwartz Integral: Integration with respect to a $\sigma$-additive Measure, Birkhäuser Verlag, Basel-BerlinBoston, 2008.

[26] P. Pérez Carreras and J. Bonet, Barrelled Locally Convex Spaces, North Holland, Amsterdam, 1987.

[27] W. Wnuk, Locally solid Riesz spaces not containing $c_{0}$, Bull. Polish Acad. Sci. Math. 36 (1988), 51-56.

[28] K. Yosida, Functional Analysis, Springer Verlag, Berlin-Heidelberg, 1965.

[29] A.C. Zaanen, Riesz Spaces II, North Holland, Amsterdam, 1983.

[30] R. Zaharopol, Mean ergodicity of power bounded operators in countably order complete Banach lattices, Math. Z. 192 (1986), 81-88.

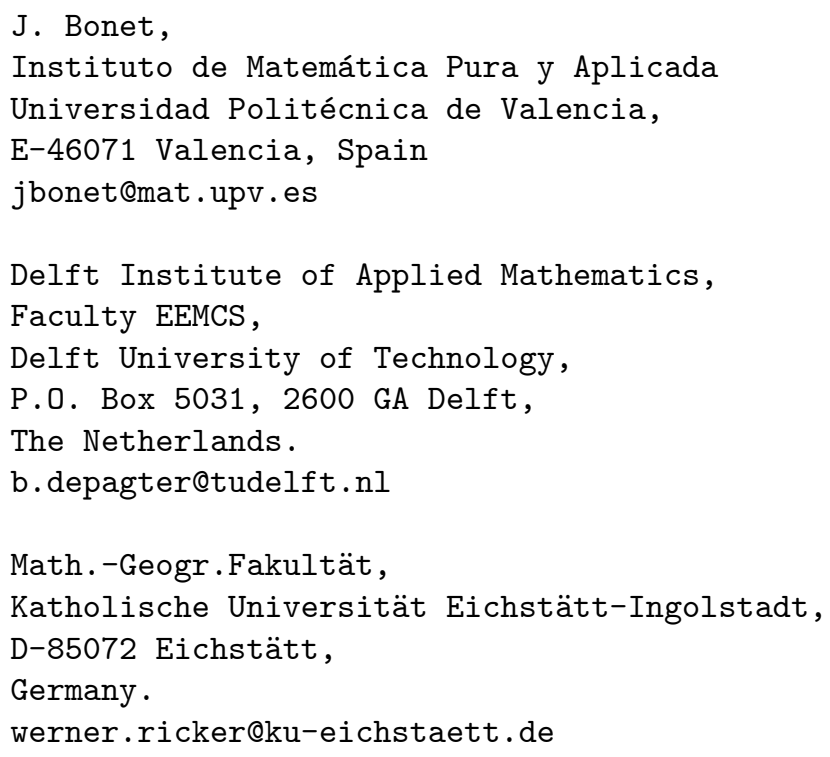

